\title{
In welchen Kantonen ist die Führung einer Arztpraxis in der Rechtsform einer Kapitalgesellschaft möglich?
}

Bis vor wenigen Jahren entsprach es den allgemeingültigen gesetzlichen Regeln, dass Ärzte ihren Beruf als selbständige Tätigkeit ausübten. Daraus folgte, dass Arztpraxen in der Vergangenheit regelmässig als Einzelfirma oder Personengesellschaft geführt wurden. Erst der - wenn auch nicht kantonsübergreifende - Verständniswandel des Begriffs der selbständigen Erwerbstätigkeit und vereinzelte Gesetzes- und/oder Praxisänderungen haben den erforderlichen Raum geschaffen, um in der Mehrheit der Kantone Arztpraxen auch in Form einer Kapitalgesellschaft (Aktiengesellschaft/AG, Gesellschaft mit beschränkter Haftung/ $\mathrm{GmbH}$ ) betreiben zu können.

Anna-Maria Willi, Adrian Schmid

Korrespondenz: Anna-Maria Willi, lic. iur., Rechtsanwältin Balmer-Etienne AG Kauffmannweg 4 CH-6003 Luzern Tel. 0412281111 Fax 0412281100 anna-maria.willi@balmer-etienne.ch

\section{Einleitung}

Gesetzes- und Praxisänderungen, aber auch der Verständniswandel des Begriffs der selbständigen Erwerbstätigkeit, haben in mehreren Kantonen die Möglichkeit eröffnet, Arztpraxen als Kapitalgesellschaften zu führen. Diese Öffnung entspricht dabei dem verbreiteten Wunsch vieler medizinischer Leistungserbringer, ihre Praxis als Aktiengesellschaft oder $\mathrm{GmbH}$ zu führen. Ursprung dieses Wunsches sind in der Regel die Vorteile, die eine Kapitalgesellschaft mit sich bringt. So wird einerseits ein vernetztes, disziplinenübergreifendes Dienstleistungsangebot ermöglicht. Anderseits führt diese Gesellschaftsform bei entsprechender Ausgestaltung aber auch zu einer anteilsmässigen Kosten- bzw. Auslagenreduktion. Individuellere Gestaltungsmöglichkeiten in der Gesellschaftsorganisation, die Zulässigkeit, Zweigniederlassungen zu gründen, und die Chance auf eine Optimierung der Steuerbelastung sind weitere Pluspunkte einer als Kapitalgesellschaft geführten Praxis. Zudem bedeutet eine kapitalgesellschaftlich organisierte Praxis auch eine einfachere Übertragbarkeit derselben. Dies kann sich insbesondere bei einer Nachfolgeregelung oder bei mehreren Beteiligten vorteilhaft auswirken. Nicht zuletzt wird bei wirtschaftlichen Misserfolgen das Haftungssubstrat grundsätzlich auf das eigentliche Gesellschaftsvermögen beschränkt. Eine weitergehende Haftung statuiert aber beispielsweise Art. 9 Abs. 4 des Rahmenvertrages

\section{Dans quels cantons est-il possible} de gérer un cabinet médical sous la forme juridique d'une société de capitaux?

Ces derniers temps, la question est souvent posée de savoir s'il est admissible de gérer un cabinet médical sous la forme d'une société anonyme (SA) ou à responsabilité limitée (SàrL). La réponse à cette interrogation étant du ressort de chaque canton, il convient tout d'abord de consulter la loi cantonale concernée. Cette dernière ne contient toutefois que rarement une réglementation explicite, et il faut donc faire recours à d'autres documents pour pouvoir se prononcer définitivement. $A$ quelques exceptions près, la réponse se trouve dans la pratique que suivent les autorités cantonales en la matière. Le présent article vise donc notamment à donner un aperçu des diverses pratiques cantonales en vigueur, afin de pouvoir déterminer plus facilement si la forme juridique d'une SA ou d'une SàrL est admissible ou non pour un cabinet médical dans tel ou tel canton. 
FMH-santésuisse, welcher eine solidarische Haftung für Vertragsverletzungen der in einer juristischen Person arbeitenden Ärzte vorsieht, sofern diese unter einer einzigen Registernummer tätig sind. Da die santésuisse seit geraumer Zeit die Registernummern nur noch einzelnen Ärzten erteilt, kann dieser Artikel im heutigen Zeitpunkt für eine Haftungsverschärfung nicht herangezogen werden. Dies insbesondere auch vor dem Hintergrund, dass die rechtliche Durchsetzbarkeit dieser Bestimmung an sich nicht unbestritten ist.

Vorliegend soll nun aufgezeigt werden, welche gesetzlichen - eidgenössischen oder kantonalen - Grundlagen in die Beantwortung der Frage nach der Zulässigkeit der Führung einer Arztpraxis in der Form einer Kapitalgesellschaft einfliessen. Zudem soll aber auch die weit bedeutendere kantonale Behördenpraxis beleuchtet werden, die mehrheitlich abschliessend entscheidet, ob eine medizinische Kapitalgesellschaft im jeweiligen Kanton als zulässig erachtet wird.

\section{Gesetzliche Grundlage}

Die Zulässigkeit des Betriebes einer Arztpraxis im Kleid einer Kapitalgesellschaft richtet sich zunächst nach den gesetzlichen Grundlagen. Im eidgenössischen Recht ist insbesondere das Krankenversicherungsgesetz (KVG) zu berücksichtigen. So sieht Art. 36 f. KVG ausdrücklich die Möglichkeit vor, als angestellter Arzt ausserhalb des Spitalbetriebs tätig zu sein. Mangels anderslautender eidgenössischer Normen ist eine Leistungserbringung innerhalb einer Kapitalgesellschaft folglich zulässig. Diese Möglichkeit bleibt aber so lange eine theoretische, als dass die Erteilung der jeweiligen Berufsausübungs- und Praxisbewilligung des einzelnen medizinischen Leistungserbringers kantonal geregelt ist. So kann jeder Kanton für sich entscheiden, ob er einem Arzt eine Praxisbzw. Betriebsbewilligung zur Führung einer Kapitalgesellschaft ausstellen will. Die Kantone sind der Regelung dieser Bewilligungsvoraussetzungen in einem formellen Gesetz jedoch weder vollumfänglich noch einheitlich nachgekommen. Drei Viertel aller deutschsprachigen Kantone sehen in ihren kantonalen Gesundheitsgesetzen gar keine Regelung vor, einige wenige verbieten bzw. bewilligen einen solchen Zusammenschluss explizit und wieder andere lassen einen solchen $\mathrm{Zu}$ sammenschluss durch Auslegung ihrer Gesetze praxisgemäss zu bzw. verbieten einen solchen. In Kenntnis der fehlenden gesetzlichen Grundlage sind gegenwärtig einige wenige Kantone (AG, NW, OW, SG, ZH und ZG) dabei, ihr kantonales Gesundheitsgesetz zu revidieren oder haben ein revidiertes Gesetz eben in Kraft gesetzt. Ob nach der Gesetzesrevision der Betrieb einer Arztpraxis in Form einer Kapitalgesellschaft zulässig sein wird, ist im heutigen Zeitpunkt jedoch noch mehrheitlich ungewiss.

\section{Standesrecht}

Doch selbst für den Fall, dass sich die kantonale gesetzliche Grundlage nicht gegen den Zusammenschluss von Ärzten in einem Unternehmen mit juristischer Persönlichkeit ausspricht, bedeutet dieses Fehlen noch keine Garantie, dass eine derartige Verbindung voraussetzungslos möglich ist. Neben der gesetzlichen Grundlage könnte zur Beantwortung der Zulässigkeit vielmehr auch ein entsprechendes Standesrecht eine entscheidende Rolle spielen. So sprach sich beispielsweise das Standesrecht der Schweizerischen Zahnärztegesellschaft (SSO) bis zum 1. Oktober 2007 explizit gegen die Möglichkeit der Führung einer Praxis in der Rechtsform einer juristischen Person aus. Im Gegensatz dazu enthält die überkantonale Standesordnung der Verbindung der Schweizer Ärztinnen und Ärzte (FMH) kein Verbot, den Arztberuf als angestellter Arzt auszuüben. Vielmehr hat die FMH als Dachorganisation der Schweizerischen Ärzteschaft die Rechtsform der Arztpraxis bewusst offen gelassen. Dies einerseits, um zu gewährleisten, dass die FMH-Standesordnung für alle Mitglieder der FMH anwendbar ist, und anderseits, um angestellte Ärzte - beispielsweise in HMO-Praxen - nicht in eine standesrechtliche Illegalität abzudrängen. Mangels entsprechender Auflagen der FMH-Standesordnung hinsichtlich der Führung einer medizinischen Kapitalgesellschaft wird der Gestaltungsfreiraum der Mitglieder der FMH standesrechtlich somit nicht eingeschränkt. Im Zusammenhang mit der Praxisorganisation sind gleichwohl einige wenige standesrechtliche Auflagen zu beachten, die die Rechtssicherheit der Patienten sicherstellen sollen, wie die Betreuungskontinuität, eine ausreichende Haftpflichtversicherung und Klarheit hinsichtlich des Behandlungsvertrages.

\section{Behördenpraxis}

Wie vorgängig bereits erwähnt, kennen die wenigsten Kantone eine gesetzliche Regelung, die sich zur Frage der Zulässigkeit der Führung einer medizinischen Kapitalgesellschaft verbindlich äussert. Dies führt dazu, dass der Auslegung der kantonalen Gesetze bzw. der Beachtung der kantonalen Behördenpraxis grösste Bedeutung zukommt. Weshalb sich Kantone dabei für oder gegen die Führung einer Arztpraxis als Kapitalgesellschaft aussprechen, hat ihren Ursprung grösstenteils im unterschiedlichen Verständnis der gesetzlichen Norm, welche besagt, dass die 


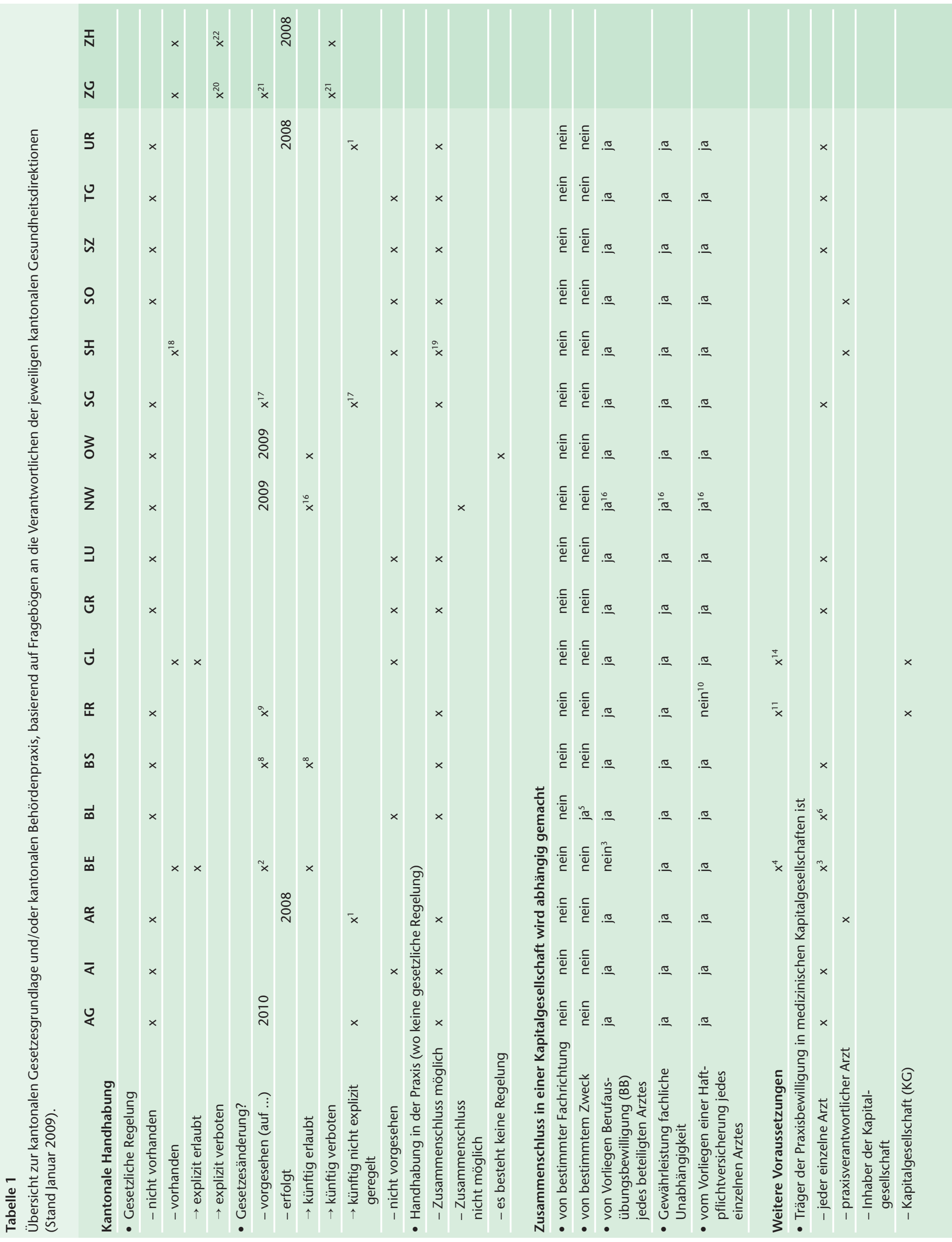




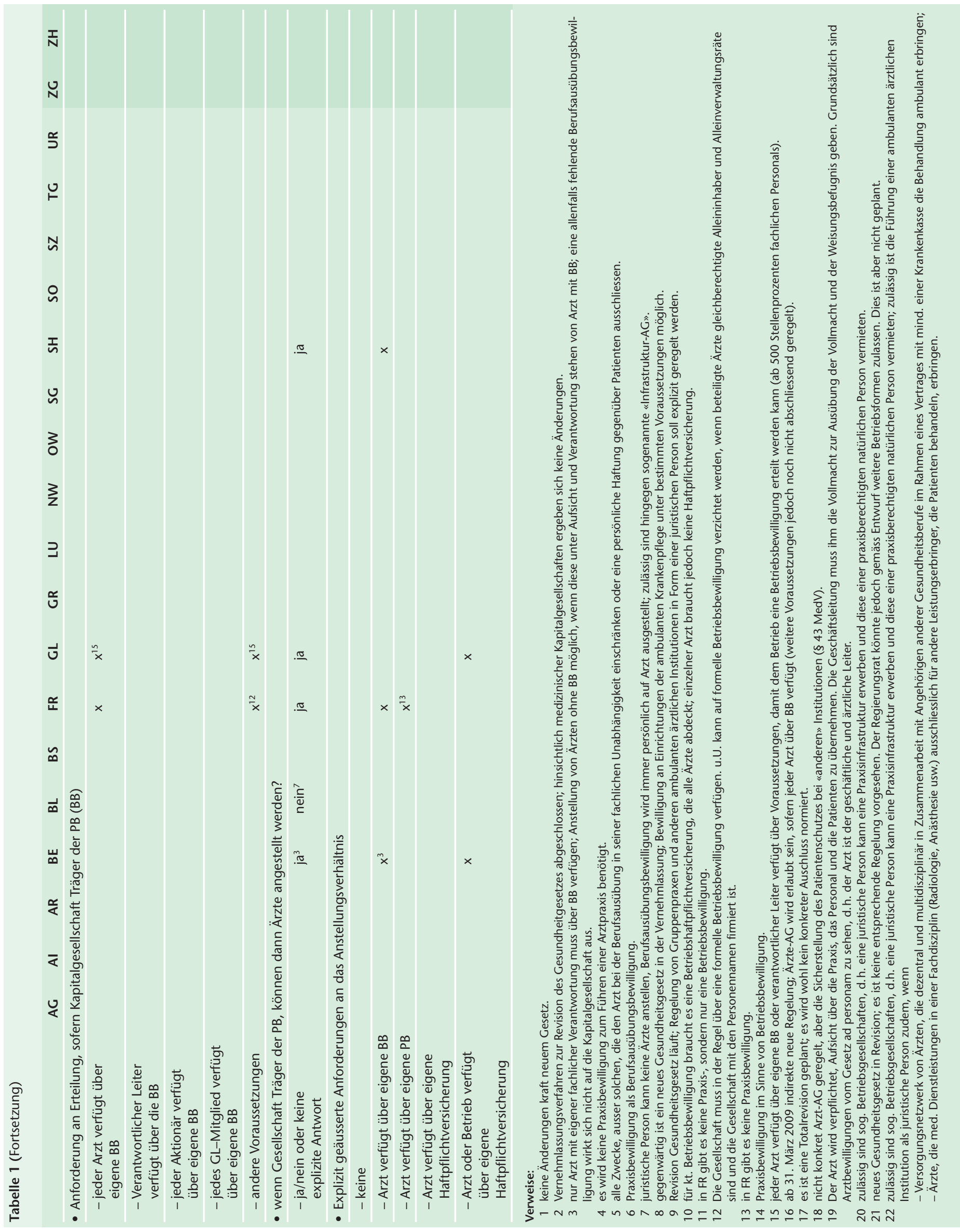


ärztliche Tätigkeit als selbständige Erwerbstätigkeit auszuüben ist. Weiter werden aber auch das Schutzbedürfnis der Patienten, die aufwendigere Kontrolle der Ärzte und eine allfällige fehlende Transparenz hinsichtlich der Gesellschaft als Begründung für ein Verbot vorgebracht. Diese unterschiedliche und uneinheitliche Behördenpraxis macht schnelle Abklärungen im Vorfeld einer konkret beabsichtigten Gesellschaftsgründung äusserst schwierig. Dies umso mehr, als gewisse kantonale Behörden gegenwärtig eine Praxisänderung in Betracht ziehen und deshalb keine abschliessenden Auskünfte erteilen können.

Im Wissen um diese bestehende Problematik sind wir der Frage nach der jeweiligen kantonalen Zulässigkeit nachgegangen. So haben wir bei allen Deutschschweizer Kantonen entsprechende Abklärungen vorgenommen und Umfragen mittels Fragebögen getätigt. Die daraus fliessenden Erkenntnisse wurden in eine schematische Aufstellung (Tab. 1) übernommen, bei der wir uns aufgrund des beabsichtigten Übersichtscharakters auf einige wenige Punkte beschränken mussten. Insbesondere war es uns nicht möglich, alle kantonalen Ausnahmen und Ergänzungen in der Übersicht aufzuzeigen. Die Übersicht zeigt hingegen auf, welche Kantone eine medizinische Kapitalgesellschaft zulassen und welche nicht bzw. auf welche Grundlagen sich diese Aussage stützt. Zudem werden auch Fragen nach der Ausgestaltung der Bewilligungserteilung aufgegriffen.

\section{Fazit}

Zur Beantwortung der Frage nach der Zulässigkeit der Führung einer Arztpraxis in Form einer Kapitalgesellschaft kommt, aufgrund der vorangegangenen Erläuterungen, insbesondere der kantonalen Behördenpraxis grosses Gewicht zu. Hat sich ein Kanton, sei es per Gesetz oder Behördenpraxis, erst einmal für die Zulässigkeit einer medizinischen Kapitalgesellschaft ausgesprochen, so empfiehlt es sich im Vorfeld einer beabsichtigten Gründung, unter anderem folgende Themen näher zu beleuchten, um anschliessend entscheiden zu können, ob eine Gründung tatsächlich sinnvoll und zweckmässig erscheint:

- Vorteile einer kapitalgesellschaftlich geführten Arztpraxis;

- Gründungsprozedere bzw. -art (bspw. Gründung mittels Sacheinlage);

- Gründungskosten;

- Erteilung der ZSR-Nummer;

- Frage der Anwendbarkeit des Medizinalberufegesetzes;

- allfällige Anwendbarkeit des Binnenmarktgesetzes;

- Aktionärbindungsverträge;

- Aspekte des Haftpflichtrechts;

- Aspekte des Steuerrechts - entstehen durch die Gründung steuerliche Vorteile?

- Verantwortlichkeit der Ärzte gegenüber Patienten und Versicherern.

\section{Literatur}

1 Weber RH, Etter B (Hrsg.). Die medizinische Aktiengesellschaft - Rechtsfragen der Unternehmensorganisation und der Unternehmensführung. Bern: Stämpfli; 2007.

2 Schaffhauser R, Kieser U, Poledna T (Hrsg.). Das neue Medizinalberufegesetz. Schriftenreihe des Instituts für Rechtswissenschaft und Rechtspraxis. Band 49. St. Gallen: Universität; 2008.

3 Kuhn MW, Poledna T (Hrsg). Arztrecht in der Praxis. Zürich: Schulthess; 2007. 